\title{
Optimization of Routing in Distributed Sensor Networks Using Heuristic Technique Approach
}

\author{
Lokesh. B. Bhajantri *, Nalini. $\mathrm{N}^{* *}$ \\ *Department of Information Science and Engineering, Basaveshwar Engineering College, Bagalkot, Karnataka, India. \\ **Department of Computer Science and Engineering, Nitte Meenakashi Institute of Technology (NMIT), Bangalore. \\ lokeshcse@yahoo.co.in, nalinaniranjan@hotmail.com
}

\begin{abstract}
Distributed Sensor Network consists set of distributed nodes having the capability of sensing, computation and wireless communications. Power management, various routing and data dissemination protocols have been specifically designed for DSN, where energy consumption is an essential design issues for routing. Optimization of routing method is an essential for routing of DSN because of long communication distances between distributed sensor nodes and sink node in a network can greatly drain the energy of sensors and decrease the lifetime of the network.

In this paper, simulation is carried out for optimization of routing in DSNs using MATLAB software. The objective is to maximize the network life time and improve the energy efficiency using heuristic technique. A proposed Genetic Algorithm based routing protocol is used for solving an optimization through the evolution of genes parameters, which are coded by strings of characters or numbers and genetic operations (selection, crossover and mutation) are iterated. Finally, the performance parameters for the proposed scheme are evaluated and are shown in terms of energy and routing efficiency, time computation and network lifetime.
\end{abstract}

Index Terms - Distributed Sensor Networks (DSNs), Genetic Algorithm (GA), Routing, Selection, Crossover and Mutation

\section{INTRODUCTION}

A DSN has a set of distributed sensor nodes that are interconnected by a communication network in the environment. The sensors are deeply embedded devices that are integrated with a physical environment and capable of acquiring signals, processing the signals, communicating, context aware computing and performing computation tasks. While this new class of networks has the potential to enable wide range of applications, it also poses serious challenges like routing, data gathering and dissemination, frequent network topology changes, limited computational, memory, power supply and sensors are more prone to failures. With all these constraints an efficient and effective method to extract data from the network is challenging task. DSN provides opportunity for many exciting applications, it also poses many new challenges compared with traditional networks as follows: should be power-aware, energy efficient, exception free and unattended operation or is self configuring, should operate and must respond to very dynamic environment, should be data-centric, data concentrated and application-specific [1, 2].

The routing is attempted to discover the route for data transmission from sources to sink node. The optimization of routing is an important parameter in critical applications like military, environment monitoring, disaster management, etc., where routing of data is significant. Some of routing challenges and design issues in DSN as follows: Network dynamics, node deployment, energy considerations, data delivery models, node capabilities, data aggregation/fusion. Many routing protocols are considered in DSN environment as follows: Sensor Protocols for Information via Negotiation (SPIN), Low-Energy Adaptive Clustering Hierarchy (LEACH), Geographic and Energy Aware Routing (GEAR), and Greedy Perimeter Stateless Routing (GPSR) [3, 4].

The paper is organized is as follows: Section II gives brief description of related work for proposed system. Section III presents an overview of proposed GA. Section IV presents a proposed work. Simulation model is presented in Section V. Finally, Section VI concludes the paper.

\section{RELATED WORKS}

Some of the related works on routing and heuristic techniques are as follows: The work given in [5] presents energy efficient routing protocol in wireless sensor networks. This paper proposes a new data transfer protocol employed in large-scale wireless sensor network. Here, only one designated cluster-head sends the data received from other cluster-heads to the base station during one round of communication. Also, adopt a method that replaces weak cluster-head using a proxy node.

An optimization scheme for wireless sensor network localization is presented in [6]. This paper provides an overview of centralized distance based algorithms for estimating the positions of nodes in a sensor network. The work presented in [7] describes a survey on routing protocols for wireless sensor networks. This paper surveys recent routing protocols for sensor networks and 
presents a classification for the various approaches pursued. The work presented in [8] describes maintenance efficient routing in wireless sensor networks. This paper presents an analysis framework of routing protocols that can be applied to produce sensor fields that are much less expensive to maintain. The framework is based on a maintenance model that is simple, yet flexible enough to capture real world deployment scenarios of sensor networks.

The technique for efficient routing in wireless sensor network is given in [9]. Wireless sensor network is a tiny sensor device about a cubic size having sensors and small battery, which enables applications that connect the physical world with pervasive networks. These sensor devices do not only have the ability to communicate information across the sensor network, but also to cooperate in performing more complex tasks, like signal processing, data aggregation and compression in the network rather than out of the network. Various routing protocols have been designed and developed for wireless sensor networks because the routing in wireless sensor network is distinguished from other networks.

The work given in [10] describes optimization approaches to dynamic routing of measurements and models in a sensor network object tracking problem. Inter-sensor communication often comprises a significant portion of energy expenditures in a sensor network as compared to sensing and computation. It discusses an integrated approach to dynamically routing measurements and models in a sensor network. Specifically, it examine the problem of tracking objects within a region wherein the responsibility for combining measurements and updating a posterior state distribution is assigned to a single sensor at any given time step. It proposes an algorithm based on a dynamic programming roll-out formulation of the minimum cost problem.

The work presented in [11] describes energy aware routing algorithm for wireless sensor network. Technological advancements made major research challenges in the area of wireless sensor network. Power saving is the foremost criteria in this field. Consumption of energy is considerably reduced on properly designed protocol. This paper focuses on energy aware fuzzy based routing protocol.

The work in [12] describes a dynamic routing control based on a genetic algorithm. This paper demonstrates that a dynamic routing control based on a genetic algorithm can provide flexible real-time management of dynamic traffic changes in broadband networks. It proposes a string structure, each of whose elements represents paths between each pair of origin and destination terminal nodes, and a new technique using the past solutions as the initial data for new searches. These techniques dramatically improve the efficiency and convergence speed of the genetic algorithm.

The description of genetic algorithm for hierarchical wireless sensor networks is in [13]. Large scale wireless sensor networks (WSNs) can be used for various pervasive and ubiquitous applications such as security, health-care, industry automation, agriculture, environment and habitat monitoring. As hierarchical clusters can reduce the energy consumption requirements for WSNs, investigate intelligent techniques for cluster formation and management. A GA is used to create energy efficient clusters for data dissemination in wireless sensor networks. The simulation result shows that the proposed intelligent hierarchical clustering technique can extend the network lifetime for different network deployment environments.

The work presented in [14] describes GA inspired routing protocol for wireless sensor networks. The key parameters that need to be addressed while designing protocols for sensor networks are its energy awareness and computational feasibility in resource constrained sensor nodes. Variation in the distances of nodes from the base station and differences in inter-nodal distances are primary factors causing unequal energy dissipation among the nodes. Thus energy difference among the nodes increases with time resulting in degraded network performance. This paper proposes a Genetic algorithm inspired Routing Protocol (GROUP) which shows enhanced performance in terms of energy efficiency and network life time over other schemes.

The work presented in [15] describes genetic algorithm based efficient routing scheme for multicast networks. Due to the advent of many new multimedia applications in high speed networks, the issue of multicast routing has become more and more important. This paper proposes a new multicast routing scheme based on GA. Some of the related works are given in [16, 17, 18, 19, 20, 21, and 22].

A lot of study has been done in the area of energy efficient routing for WSNs. Earlier work do not consider the optimization of routing in DSN environment in terms of energy efficiency, network lifetime and time complexity. Researchers shows that the cluster based algorithms perform better compared to flat algorithms using GA. In this work, we have proposed GA inspired routing protocol for optimization of routing over the scheme. The discovering the shortest paths from the source nodes to the sink node for optimization of routing to increase the efficiency of the network in terms of network lifetime and energy efficiency. And also we have considered two point crossover processes in this scheme but earlier works considers only one point crossover, which is less efficient.

The following are the important assumptions made in the proposed scheme.

- Nodes are stationary and deployed in a random fashion in DSN environment.

- It is assumed that all nodes in the network are static and have initial energy.

- $\quad$ All nodes are equipped with GPS. During set up phase all nodes convey its location and energy information to the BS.

- $\quad$ Nodes sense the data periodically and send it to the sink node with multi-hop communication over the network using GA inspired routing protocol.

\section{Proposed Heuristic TeChNIQUE}


A GA is a search heuristic method that mimics the process of natural evolution. This heuristic is routinely used to generate useful solutions to optimization and search problems. GA belong to the larger class of evolutionary algorithms, which generate solutions to optimization problems using techniques inspired by natural evolution, such as inheritance, selection, crossover, and mutation [23]. In a GA, a population of strings (called chromosomes or the genotype of the genome) which encode candidate solutions (called individuals, creatures, or phenotypes) to an optimization problem evolves toward better solutions. Traditionally, solutions are represented in binary as strings of 0 's and 1's, but other encodings are also possible. In proposed work, node based encoding method is used.

The evolution usually starts from a population of randomly generated individuals and happens in generations. In each generation, the fitness of every individual in the population is evaluated, multiple individuals are stochastically selected from the current population (based on their fitness) and modified (recombined and possibly randomly mutated) to form a new population. The new population is then used in the next iteration of the algorithm. Commonly, the algorithm terminates when either a maximum number of generations has been produced or a satisfactory fitness level has been reached for the population. When the algorithm is terminated, once may have got the optimal solution.

\section{A. Genetic Algorithm Operations}

Some of the operations used in the proposed GA are as follows:

Genetic Representation: A chromosome is a collection of genes and each chromosome represents a number of nodes or genes in path for a given network. In this work, a chromosome of the proposed GA consists of sequences of positive integers that represent the ID's of nodes. The variable length of the chromosome is represented as the total number of nodes or genes in the path for network. The length of the chromosome should not exceed the maximum length i.e total number of nodes in the network.

Population: A population (P) is a collection of chromosomes, where a family of ' $\mathrm{r}$ ' chromosomes is represented as $\mathrm{P}=\left(\mathrm{C}_{1}, \mathrm{C}_{2} \ldots \mathrm{C}_{\mathrm{r}}\right)$. In which as the number of nodes increases, then size of the population increases in the network. In this work, selection of two chromosomes is based on roulette wheel selection for finding optimal path in the network.

Fitness function: Each chromosome is evaluated by a function known as the fitness function, which is usually called the cost function or the objective function of the corresponding optimization problem. Fitness is the core part of a GA. The proposed fitness function is designed to increase the lifetime of the system, which evaluates whether a particular chromosome increases lifetime or not. We always preserve the historically obtained best chromosome i.e., with the highest fitness value. Fitness function assigns a real value to each path those function consists of parameters which needs to be optimized. In this work, the fitness function is measured on the basis of energy and bandwidth of each node. The fitness function of the proposed scheme is formulated as follows:

$$
\left.\mathbf{F}_{\mathbf{F u n}}=\underset{\mathbf{i}=\mathbf{l}}{\stackrel{\mathrm{N}}{\Sigma} \mathrm{E}_{\mathrm{N}}} * \underset{\mathbf{i}=\mathbf{l}}{\mathrm{N}} \mathrm{BW} \underset{\mathbf{i}=\mathbf{l}}{\mathrm{N}} \mathrm{C}_{\mathrm{N}}\right)^{*} \text { Path }
$$

Where,

$$
\begin{aligned}
& \mathrm{F}_{\text {Fun }}=\text { Fitness Function } \\
& \mathrm{E}_{\mathrm{N}}=\text { Energy of each node } \\
& \mathrm{C}_{\mathrm{N}}=\text { Cost of node } \\
& \mathrm{BW}=\text { Bandwidth of each node }
\end{aligned}
$$

Path is a variable, which depends upon whether there is an existing path between source and destination or not.

Selection: Selection process is used to select two parent chromosomes which are having highest fitness value. This selection process can be implemented by many algorithms such as tournament selection, rank selection, steady state selection and roulette wheel selection. In proposed work we have used roulette wheel selection method it gives contribution of each chromosome to the total fitness value.

Crossover: In this work, we have used two point crossover method. Crossover process exchanges the genes of two parent chromosomes. Choose a crossover point and from that point cross the parent path. First part of first parent is exchanged with first part of second parent. Similarly, second part of the first parent is exchanged with second part of second parent. We have considered the probability of crossover is in between 0.6 and 1.0 .

Mutation: In this work, we have considered single point mutation process, in which generate random value between 1 to $\mathrm{N}$ where, ' $\mathrm{N}$ ' is number of nodes. Replace this random value in random position. These two mutated C1 \& C2 are added to population by removing the two lower fitness valued paths to form new generation.

Selection, crossover and mutation operations are iterated until it meets the termination criteria condition.

\section{PROPOSED WORK}

The proposed GA inspired routing protocol which shows enhanced performance in terms of energy efficiency and network lifetime over the schemes by discovering the shortest paths from the source nodes to the base node or sink node. A multi-path data transfer is also accomplished to provide reliable network operations by considering the energy levels of the nodes. In this section, we describe system model for proposed scheme and functioning scheme of proposed work. 


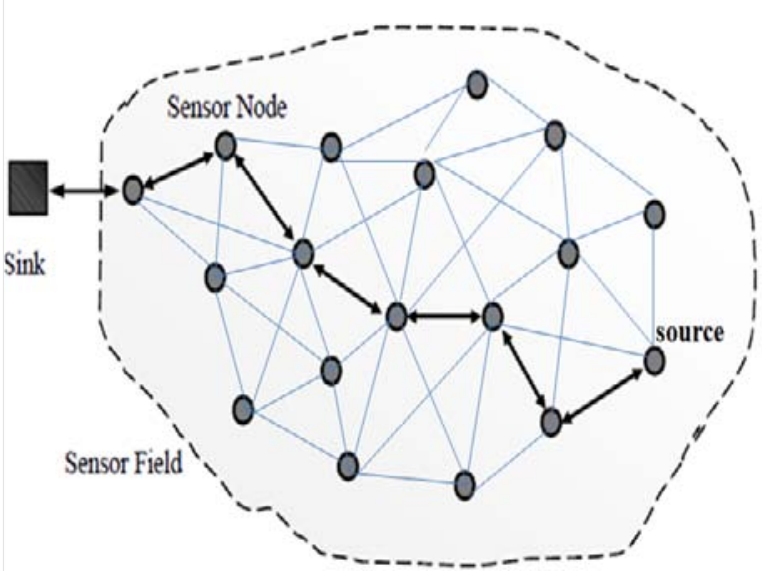

Figure 1: System Architecture.

The proposed system architecture consists of set of sensor nodes $S=\left\{S_{1}, S_{2}, S_{3}, S_{4},--S_{n}\right\}$, which are placed randomly as shown in Fig. 1. A node senses the data periodically and sends it to the sink node with multi - hop communication. It is assumed that all nodes (sensor and sink nodes) in the network are static and have fixed initial energy $\left(\mathrm{I}_{\mathrm{E}}\right)$. It is also assumed that during deployment phase each node have full energy. Sensor nodes are computing the optimization of routing in the DSN environment. The operation sequence of the proposed scheme is as follows: 1) Deploy the number of sensor nodes as randomly in DSN environment. 2) Sensor nodes receive the neighbor nodes information in the network with the help of GPS. 3) To generate the population size for the network. 4) To select the two parent paths or chromosomes based on roulette wheel selection method. 5) To apply genetic operations such as: crossover and mutation operations and 6) Sink node takes the action upon receiving the information form sensor nodes.

\section{A. Functioning scheme}

In this section, functions of GA and corresponding algorithms are explained. Some of the notations used in description of the scheme are given in table 1.

TABLE 1: Notations

\begin{tabular}{|l|l|}
\hline \multicolumn{1}{|c|}{ Symbols } & \multicolumn{1}{c|}{ Definitions } \\
\hline$N=\left\{\mathrm{S}_{1}, \mathrm{~S}_{2}, \mathrm{~S}_{3},----\mathrm{S}_{\mathrm{n}}\right\}$ & Set of Sensor Nodes \\
\hline $\mathrm{I}_{\mathrm{E}}$ & Initial Energy of sensor nodes \\
\hline $\mathrm{C}_{\mathrm{SN}}$ & Costs between each sensor nodes \\
\hline $\mathrm{THL}_{\mathrm{EN}}$ & Threshold Level Energy \\
\hline $\mathrm{E}_{\mathrm{tr}}$ & Total energy consumed by path \\
\hline $\mathrm{T}_{\mathrm{f}}$ & Total fitness \\
\hline $\mathrm{A}_{\mathrm{f}}$ & Average fitness \\
\hline $\mathrm{C}_{\mathrm{f}}$ & Cumulative fitness \\
\hline $\mathrm{C}_{\mathrm{p}}$ & Crossover probability condition \\
\hline $\mathrm{C}_{1}, \mathrm{C}_{2}, \mathrm{C}_{3}, \mathrm{C}_{4},------\mathrm{C}_{\mathrm{N}}$ & Set of Childs \\
\hline $\mathrm{P}_{1}, \mathrm{P}_{2}, \mathrm{P}_{3}, \mathrm{P}_{4},------\mathrm{P}_{\mathrm{N}}$ & Set of Parents \\
\hline $\mathrm{R}_{1}, \mathrm{R}_{2}, \mathrm{R}_{3},----------\mathrm{R}_{\mathrm{N}}$ & Set of Random Numbers \\
\hline $\mathrm{D}$ & Destination Node \\
\hline $\mathrm{M}_{\mathrm{P}}$ & Mutation probability condition \\
\hline $\mathrm{F}(\mathrm{I})$ & Fitness of I ${ }^{\text {th }}$ path \\
\hline $\mathrm{A}_{(\mathrm{I})}$ & Average fitness of I ${ }^{\text {th }}$ paths \\
\hline $\mathrm{F}_{\mathrm{Fun}}$ & Fitness Function or Array \\
\hline $\mathrm{E}_{\mathrm{S}}$ & Energy required for sensing of each \\
\hline
\end{tabular}

\begin{tabular}{|l|l|}
\hline & node \\
\hline$E_{T}$ & $\begin{array}{l}\text { Energy required for transmission of } \\
\text { data }\end{array}$ \\
\hline$T_{E}$ & Total Energy of each node \\
\hline
\end{tabular}

B. Algorithm for Optimization of Routing in DSN Environment

Begin

1. Deploy the number of nodes in DSN Environment as randomly.

2. Initialize each sensor node properties in the environment such as: Sensor_id, Sensor_energy, and bandwidth.

3. Generate initial population which consists of randomly generated chromosomes.

4. While $\left(S_{n} !==D\right)$ Then

To check Energy; //for each node in a path If $\left(\mathrm{I}_{\mathrm{E}}<\mathrm{THL}_{\mathrm{EN}}\right)$

Considered as a dead node.

Else

Considered as an active node.

5. Evaluate the fitness of each chromosome in population size using Eq. (1) and store it in fitness function.

6. While terminating condition is not meet do

- Find the Max. Fitness form fitness function.

- If there is no change in Max. Fitness within Min. No. of iteration, break the loop.

Otherwise:

Select two parents (paths) using roulette wheel selection from population.

- Apply crossover on parents to produce two offspring's $\left(\mathrm{C}_{1}\right.$ and $\left.\mathrm{C}_{2}\right)$.

- Apply mutation on two offspring's.

- Find the least fit chromosomes (path) from population.

- If fitness of $C_{1}$ and $C_{2}$ are greater than least fit path, replace them with new $\mathrm{C}_{1}$ and $\mathrm{C}_{2}$.

End while

7. Get the chromosome with the highest fitness values from the population.

End

8. Stop.

\section{Roulette Wheel Selection}

The parent selection process of GA determines which two parents will take part in mating to create an offspring among the total participation of ' $r$ ' chromosomes. We use roulette wheel selection method, where two chromosomes are chosen randomly for mating. The roulette wheel selection method as given below:

1. Evaluate the sum of all the fitness from the fitness array.

2. Get the cumulative fitness using:
$\mathrm{C}_{\mathrm{f}}$
(I)
$=\mathrm{A}$
(I)
sum 
Where,

$\mathrm{A}(\mathrm{I})=$ fitness $(\mathrm{I})+\mathrm{A}(\mathrm{I}-1)$ and $\mathrm{I}=1,2,3,---\mathrm{n}$.

3. Generate random number $\left(\mathrm{R}_{\mathrm{N}}\right)$ between 0 and 1 .

4. Compare $R_{N}$ with all the elements of $C_{f}(I)$, if it is less than $\mathrm{C}_{\mathrm{f}}(\mathrm{I})$, then $\mathrm{i}^{\text {th }}$ chromosome is selected.

5. Repeat the step 4 to get second parent.

6. Stop.

\section{Crossover}

Crossover indicates the combination of the two parent chromosomes to produce an offspring. We have implemented the crossover method as discussed in section III.

1. Initialize crossover probability $\left(\mathrm{C}_{\mathrm{p}}\right)$.

2. Generate random number $\left(\mathrm{R}_{\mathrm{N}}\right)$ between 0 and 1 .

3. Compare $\mathrm{R}_{1}$ with crossover probability, if it is less than $\mathrm{C}_{\mathrm{p}}$ then do

- Randomly generate two crossover points on selected parents.

- Copy the nodes of $\mathrm{P}_{2}$ to $\mathrm{P}_{1}$ from first crossover point to second crossover point keeping $\mathrm{P}_{1}$ as it is from first gene to first crossover point which gives $C_{1}$.

- Copy the nodes of $\mathrm{P}_{1}$ to $\mathrm{P}_{2}$ from first crossover point to second crossover point keeping $\mathrm{P}_{2}$ as it is from first gene to first crossover point which gives $\mathrm{C}_{2}$.

Else

$$
\mathrm{C}_{1}=\mathrm{P}_{1}
$$$$
\mathrm{C}_{2}=\mathrm{P}_{2}
$$

4. Stop.

\section{E. Mutation}

Begin

1. Initialize mutation probability

2. Generate random number $R_{2}$ between 0 and 1 .

3. Compare $R_{2}$ with $M_{p}$, if it is less than $M_{p}$ then do

- Generate mutation points randomly of $\mathrm{C}_{1}$ and $\mathrm{C}_{2}$.

- Generate random numbers (replacing genes).

- $\quad$ Replace new genes at mutation points of $\mathrm{C}_{1}$ and $\mathrm{C}_{2}$ and get new mutated children. Else

$\mathrm{C}_{1}$ and $\mathrm{C}_{2}$ are taken as mutated children itself.

4. Stop.

End

\section{F. Analysis of an optimization routing in DSN}

An optimization routing in the network is analyzed in best - case and worst - case analysis are as follows:

Best-case analysis: Best-case occurs when there is an optimization of routing is directly proportional to the genetic operations (such as: population size, size of the chromosomes and number of iterations including selection, crossover and mutation). Therefore total time required for optimization of routing in best-case analysis is given by:

Time Complexity (Optimization of routing) Best-case $=\mathrm{O}$ $(\mathrm{gnm}) * \mathrm{Cp}$ and $\mathrm{Mp}=\mathrm{O}\left(\log _{\mathrm{n}}\right)$
Where,

$$
\begin{aligned}
& \mathrm{g}=\text { minimum number of generations, } \\
& \mathrm{n}=\text { the population size, } \\
& \mathrm{m}=\text { the size of the chromosomes, } \\
& \mathrm{Cp}=\text { Crossover Probability, and } \\
& \mathrm{Mp}=\text { Mutation probability }
\end{aligned}
$$

Equation (2) indicates that best-case analyses of optimization of routing, which is including minimum total cost or minimum distance between nodes and sink node and less energy consumption.

Worst-case analysis: Worst-case occurs when there is an optimization of routing is directly proportional to the genetic operation such as more number of generations or iterations, and $\mathrm{Cp}$ and $\mathrm{Mp}$ are is less than 0.6 and greater than 0.4 respectively. In this situation, considered as more time and energy is required for optimization of routing. Therefore total time required for optimization of routing in worst-case analysis is given by:

Time Complexity (Optimization of routing) Worst-case $^{=}$ $\mathrm{O}(\mathrm{gnm}) * \mathrm{Cp}$ and $\mathrm{Mp}=\mathrm{O}\left(\mathrm{nlog}_{\mathrm{n}}\right)$

Where,

$$
\begin{aligned}
& \mathrm{g}=\text { minimum number of generations, } \\
& \mathrm{n}=\text { size of the population, } \\
& \mathrm{m}=\text { size of the chromosomes, } \\
& \mathrm{Cp}=\text { Crossover Probability, and } \\
& \mathrm{Mp}=\text { Mutation probability }
\end{aligned}
$$

Equation (3) indicates that worst-case analysis of optimization of routing, which including more time and more energy consumption in the network environment.

\section{SIMULATION}

We conducted simulation of the proposed scheme by using MATLAB software. The proposed scheme has been simulated in various network scenarios. Simulation is carried out extensively with random number for 1000 iterations. This section presents the simulation model, simulation procedure, performance parameters, results and discussions.

\section{A. Simulation model}

The proposed simulation model consists of ' $N$ ' number of nodes deployed randomly in a distributed environment. Simulation is done for ' $N$ ' $\left(S_{N}=20-200\right)$ nodes used to measure the performance parameters such as energy utilization, throughput, time computation. Simulation environment comprises some of the models namely network model, channel model and battery model. These models are described as follows:

Network Model: We have considered an area of $\mathrm{l} * \mathrm{~b}$ square meters for DSN environment. Each experiment corresponds to a random placement of sensors in a fixed network area and performs the optimization of routing using GA by selecting highest fitness value for the paths based on minimum cost and less energy consumption between the nodes ( source and sink node) as it satisfies the optimization criteria. 
Channel Model: Sensors generate data packets of variable sizes. The sensing range is the same as the transmission range which is considered as an input parameter. We have considered the channel to be error free and contention between sensor nodes. All sensors have same period of periodic sensing. As for delay on a link, it can be calculated as units of time.

Battery Model: Each sensor has a battery with finite, non-replenish able energy, which was set to an initial energy in joules. Whenever a sensor transmits or receives a data packet, it consumes some energy from its battery.

The energy model for the sensors is based on the first order radio model for calculation of the energy dissipation for sensing and receiving, transmission as well as finding optimization of routing in the network. The radio can perform the power control and hence use the minimum required energy to reach the optimization criteria. It is assumed that at any given time, the value of energy required for transmitted $\left(E_{T}\right)$ and sensing $\left(E_{S}\right)$ for ' $k$ ' bit packet to another node ' $d$ ' meters is ' $I_{E}$ 'Joules for every node.

$$
\text { Total Energy }\left(\mathrm{T}_{\mathrm{E}}\right)=\mathrm{E}_{\mathrm{S}} * \mathrm{P} / \text { Bits }+\mathrm{E}_{\mathrm{T}} * \mathrm{P} / \text { Bits } * \mathrm{~d}
$$

Where,

$$
\begin{aligned}
& \mathrm{P}=\text { packet. } \\
& \mathrm{d}=\text { distance }
\end{aligned}
$$

\section{B. Simulation procedure}

Table 2 presents the simulation parameters considered for analyzing the scheme. Simulation procedure involves following steps:

Begin

1. Deploy the number of nodes randomly as in DSN environment.

2. Initialize sensor node properties.

3. Apply the proposed heuristic technique to networks.

4. Find the optimization of routing in the network.

5. Compute performance parameters of the system.

End

6. Generate graphs.

Table2: Simulation Inputs

\begin{tabular}{|l|l|l|}
\hline \multicolumn{1}{|c|}{ Parameters } & \multicolumn{1}{c|}{ Notations } & \multicolumn{1}{c|}{ Values } \\
\hline Length & $\mathrm{l}$ & 5000 meters \\
\hline Breadth node & $\mathrm{b}$ & 5000 meters \\
\hline Number of Sensor Nodes & $\mathrm{S}_{\mathrm{N}}$ & $20-200$ nodes \\
\hline $\begin{array}{l}\text { Sensor size } \\
\text { communication range }\end{array}$ & $\mathrm{MXP}_{\mathrm{S}}$ & 100 \\
\hline $\begin{array}{l}\text { Max. Population paths) } \\
\text { No.of }\end{array}$ & $\mathrm{S}_{\mathrm{T}}$ & $\begin{array}{l}\text { Roulette } \\
\text { Selection }\end{array}$ \\
\hline $\begin{array}{l}\text { Selection Type } \\
\text { Crossover Type }\end{array}$ & $\mathrm{C}_{\mathrm{T}}$ & Two point \\
\hline Crossover Probability & $\mathrm{C}_{\mathrm{P}}$ & $0.6-1.0$ \\
\hline Mutation probability & $\mathrm{M}_{\mathrm{P}}$ & $0.1-0.3$ \\
\hline Number of Iterations & $\mathrm{N}_{\mathrm{I}}$ & 1000 \\
\hline
\end{tabular}

\begin{tabular}{|l|l|l|}
\hline $\begin{array}{l}\text { Initial Energy of sensor } \\
\text { nodes }\end{array}$ & $\mathrm{I}_{\mathrm{E}}$ & 20 Joules \\
\hline Size of packets & $\mathrm{S}_{\mathrm{P}}$ & $\begin{array}{l}\text { 32, 64, 128, 256, } \\
512,1024 \text { Kbytes }\end{array}$ \\
\hline Costs between each nodes & $\mathrm{C}_{\mathrm{N}}$ & $50-100$ \\
\hline Threshold Level Energy & $\mathrm{THL}_{\mathrm{EN}}$ & $0.05 \mathrm{Joules}$ \\
\hline Min. Population size & $\mathrm{MNP}_{\mathrm{S}}$ & 25 \\
\hline $\begin{array}{l}\text { Energy required for } \\
\text { sensing of each node }\end{array}$ & $\mathrm{E}_{\mathrm{S}}$ & $50 \mathrm{~nJ} / \mathrm{Bit}$ \\
\hline $\begin{array}{l}\text { Energy required for } \\
\text { transmission of data }\end{array}$ & $\mathrm{E}_{\mathrm{T}}$ & $50 \mathrm{~nJ} / \mathrm{Bit}$ \\
\hline
\end{tabular}

\section{Performance parameters}

We have used the following parameters to measure the performance of the proposed system as:

1. Energy Consumption: It is defined as the rate of utilization of energy required for receiving and transmitting for packets across a network from source to destination (sink node). It is expressed in terms of 'mJoules'.

2. Time Computation: It is defined as the number of nodes increases, there is an increase in the time computation for routing in DSN. It is expressed in terms of 'mSeconds'.

3. Network Lifetime: It is defined as the number of nodes increases, and then network lifetime is increases in the DSN. Network Lifetime is measured in in-terms of percentage (\%ge).

4. Efficiency of routing: It is defined as the number of nodes increases, efficiency of network is decreases. It is expressed in terms of \%ge.

5. Time Taken for Routing: It is defined as the time required for routing, as the number of nodes increases, there is an increase in time of routing in the network. It is expressed in terms of 'mSeconds'.

\section{Results and Discussions}

Energy Consumption and Number of Nodes: We observe from Fig.2 that as the number of node increases, there is an increase in the energy consumption of sensor nodes in the network. By using proposed GA, sensor nodes are takes less energy consumption than without GA for routing in DSN environment. The energy consumption of sensor nodes in the network is measured in terms of 'mJoules' to perform GA. 


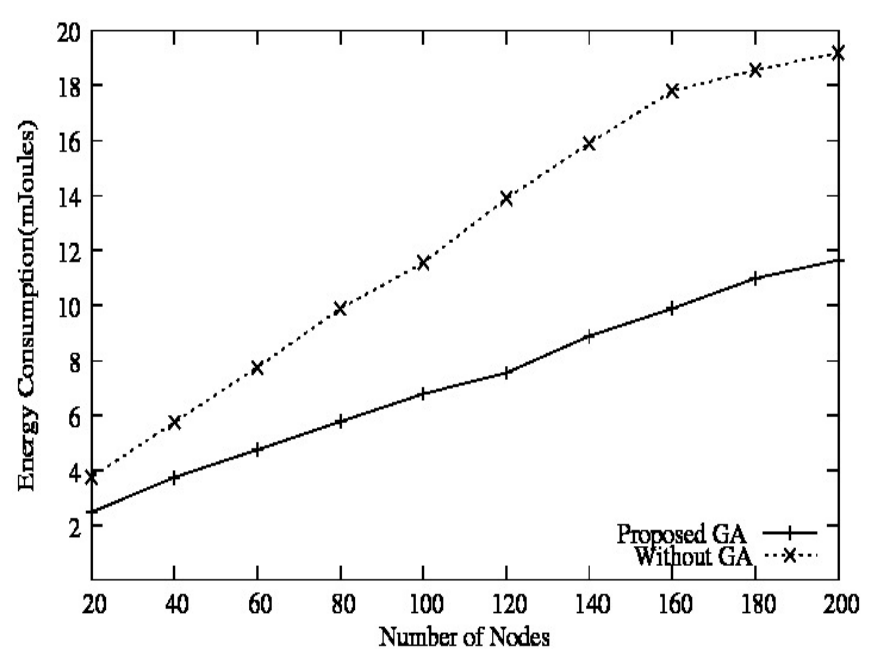

Figure 2. Energy Consumption Vs Number of Nodes.

Time Computation and Number of Nodes: We observe form Fig.3 that as the number of nodes increases, time computation of network is increases. In this, time computation is measured in terms of 'mSec'. According to proposed system, Best-case analysis occurs when there is an optimization of routing is directly proportional to the genetic operations. Therefore total time required for optimization of routing required in best-case analysis is given in equation (2), section IV.

Worst-case occurs when there is an optimization of routing is directly proportional to the genetic operations. It considered that more number of generations or iterations. Cp and Mp are less than 0.6 and greater than 0.3 respectively. In that situation, we consider, as more time and energy is required for finding an optimization of routing in the network. Therefore, total time required for optimization of routing required in worst-case analysis is given in equation (3), section IV.

Non-heuristic techniques such as Dijkstra algorithm, Bellman ford algorithms, Branch and Bound techniques etc., are not suitable for large size networks. The time complexities for these techniques are more i.e o $\left(n^{2}\right)$ [24].

Time required for routing and Number of nodes: The Fig. 4 shows that time required for routing among nodes by using proposed GA based routing scheme. As the number of nodes increases, time is increases in routing scheme. The proposed GA based routing scheme performs less time consumption than the other routing schemes in routing of data in the DSN environment.

Efficiency of routing and Number of Nodes: The Fig. 5 depicts an efficiency of routing in DSN. As the number of nodes increases, the efficiency of routing will decreases in the network. In proposed GA, efficiency of routing will be more than or equal threshold value of $75 \%$, then will be considered as the efficiency of routing in the network. Because of GA is used to solve the optimization problems in DSN. The efficiency of routing will be greater than threshold value or equal of $50 \%$ to achieve efficiency of routing in other routing networks or heuristic methods.

Network Life Time and Number of Nodes: We observe from the above Fig.6 that as the number of nodes increases, then the life time of the network will increases. In this case, the probability of crossover and mutation of the network will be in the range of $0.6-1.0$ and $0.1-0.3$ respectively and then to achieve network life time of the proposed scheme.

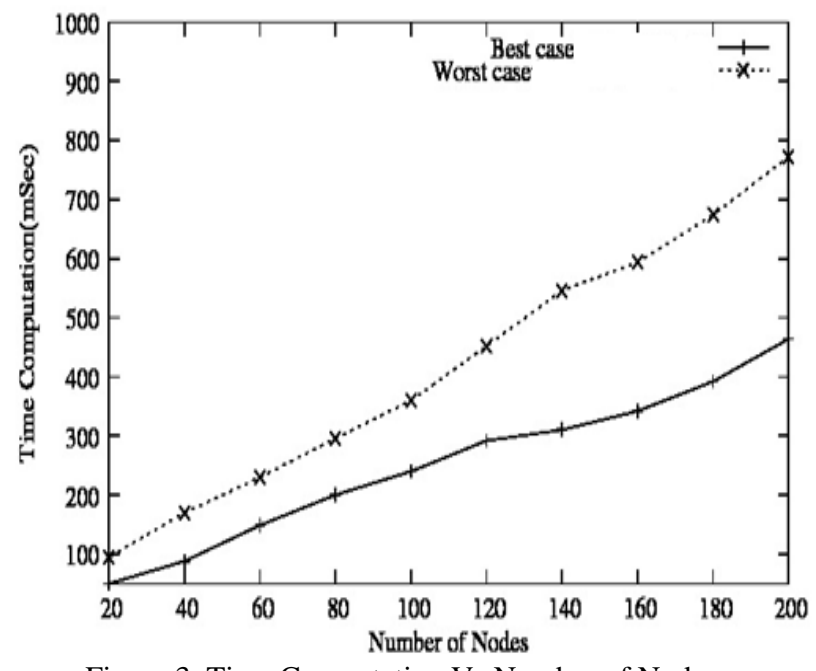

Figure 3. Time Computation Vs Number of Nodes.

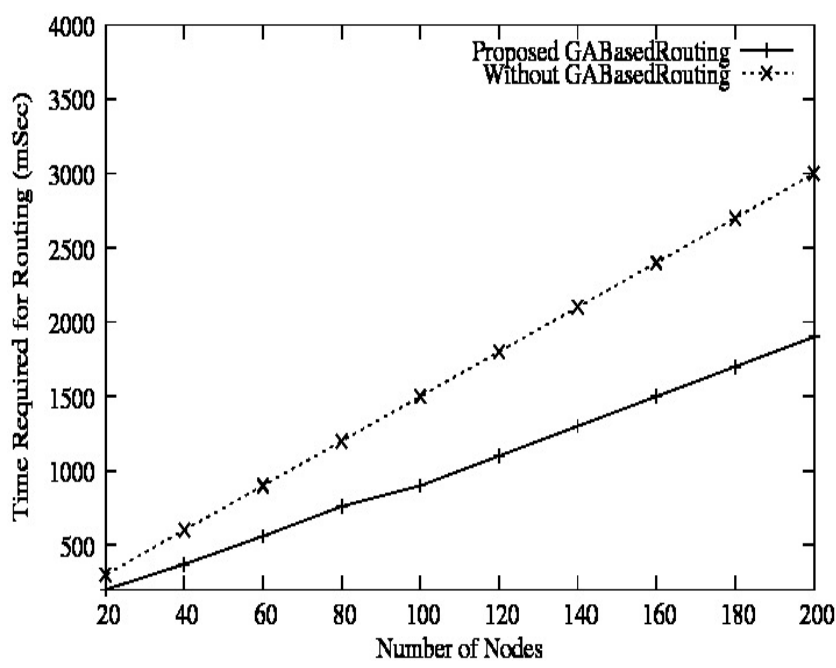

Figure 4. Time required for routing and Number of nodes.

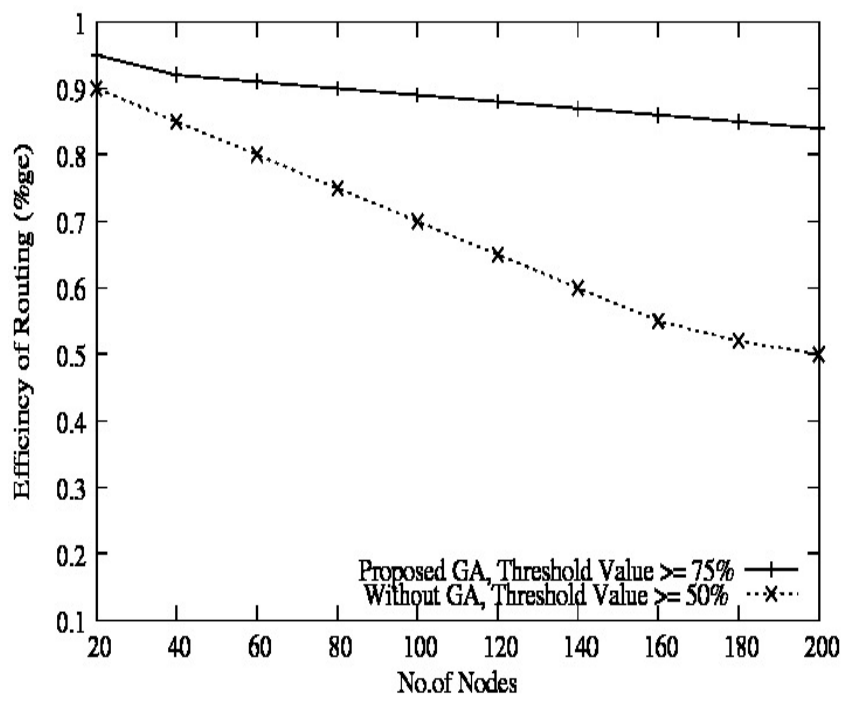

Figure 5. Efficiency of routing and Number of Nodes. 


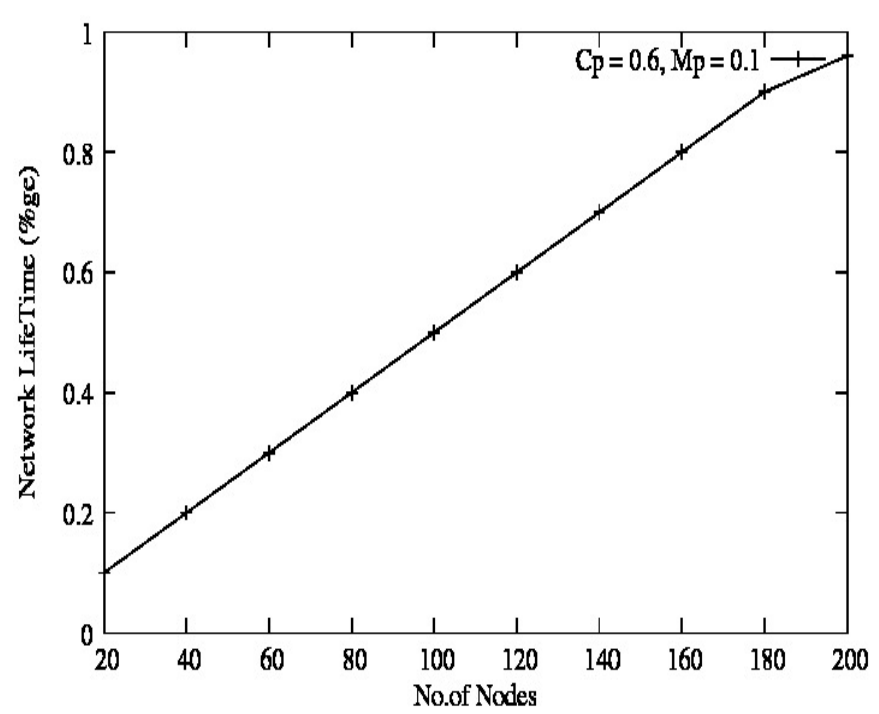

Figure 6. Network Life Time and Number of Nodes.

\section{CONCLUSION}

In this work, the proposed system model optimizes the routing in DSN. This is an efficient for finding optimal path in DSN environment. The proposed model is evaluated by comparing with performance parameters like energy utilization, efficiency, network lifetime of DSN, and time computation. Optimization of routing will have lower cost and lower energy consumption, which increases lifetime of the network. A simulation result obtained from a proposed routing algorithm is more efficient than other routing algorithms.

\section{REFERENCES}

[1] S.S.,Iyengar, Ankit. Tendon, R.R., Brooks, “A overview of Distributed Sensors Network”, Available from:

http://books.google.com/books/about/Distributed_sen sor_networks.html?id=Nff5.

[2] Shivakumar. Sastry, S.S., Iyengar, "Taxonomy of Distributed Sensors Network”, Available From: http://books.google.com/books/about/Distributed_sen sor_networks.html?id=Nff5.

[3] Heinzelman. W, Chandrakasan. A, Balakrishnan. H, "Energy-efficient Communication Protocol for Wireless Microsensor Networks". In the Proceedings of the 33rd Annual Hawaii International Conference on System Sciences, pp. 1-10, 2000.

[4] Jamal. N., Al.., Karaki, Ahmed. E., Kamal, "Routing Techniques in Wireless Sensor Networks: A Survey". IEEE wireless communications, vol.11, pp. 6-28, 2004.

[5] Kyung. Tae., Kim, Byung. Jun., Lee, Jae. Hyun., Choi, Bo. Yle., Jung, Hee. Yong., Youn, “Energy Efficient Routing Protocol in Wireless Sensor Networks”. In the Proceedings of International conference on Computational Science and Engineering, vol. 1, pp.132 - 139, 2009.
[6] Ewa. Niewiadomska., Szynkiewicz, Michal. Marks, "Optimization scheme for Wireless Sensor Network localization”. International Journal of Applied Mathematics and Computer Science, vol. 19, no.2, pp. 291-302, 2009.

[7] K. Akkaya, M. Younis, "A Survey of Routing Protocols in Wireless Sensor Networks", Journal of Elsevier Ad Hoc Network,vol. 3, no.3, pp. 325-349, 2005.

[8] Andre. Barroso, Utz. Roedig, Cormac. Sreenan, "Maintenance Efficient Routing in Wireless Sensor Networks". In the Proceedings of 2nd IEEE Workshop on Embedded Networked Sensors, pp. 97106, 2005.

[9] Keyur. M., Rana, Mukesh. A., Zaveri, “The Technique for Efficient Routing in Wireless Sensor Network". In the Proceedings of International conference on Intelligent Systems and Data Processing, pp. 1- 9, 2011.

[10] Jason. L., Williams, John. W., Fisher, Alan. S,. Willsky, "Optimization approaches to Dynamic Routing of Measurements and Models in a Sensor Network object tracking problem”. In the Proceedings of International conference on Acoustics, Speech Signal Processing, pp. 1061 -1065, 2011.

[11] S. Barani, C.Gomathy, "Energy Aware Routing algorithm for Wireless Sensor Network”. Journal of Computer Science and Engineering, vol. 2, no. 6, pp. 850-861, 2011.

[12] Shimamoto. N, Hiramatsu. A, Yamasaki. K, “A Dynamic Routing Control based on a Genetic Algorithm” In the Proceedings of IEEE International Conference on Neural Networks, vol.2, pp.1123 1128, 1993.

[13] Sajid Hussain, Abdul. Wasey., Matin, Obidul Islam, "Genetic Algorithm for Hierarchical Wireless Sensor Networks”. Journal of Networks,vol. 2, no.5, pp. 87-97 2007.

[14] Chang. Wook., Ahn, R. S., Ramakrishna,“A Genetic Algorithm for Shortest Path Routing Problem and the Sizing of Populations". IEEE Transactions on evolutionary computation, vol. 6, no. 6, pp. 566-579, 2002.

[15] Ehsan. Heidari, Ali. Movaghar, “An Efficient Method based on Genetic Algorithms to solve Sensor Network Optimization problem”. International journal on applications of graph theory in wireless ad hoc networks and sensor networks, vol.3, no.1, pp.18-33, 2011.

[16] Ayon chakraborthy, Swarup Kumar mitra, Mrinal Kanti Naskar. "A Genetic Algorithm inspired Routing protocol for Wireless Sensor Networks”. International Journal of Computational Intelligence Theory and Practice, vol, 6, no.1, pp. 1-10, 2011.

[17] Bhattacharya, R. Venkateswaran, P. Sanyal, S.K., Nandi, "Genetic Algorithm based Efficient Routing scheme for Multicast Networks”. In the Proceedings of International conference on Personal Wireless Communications, pp. 500 -504, 2005. 
[18] S. Sitharam., Iyengar, Mohan. B., Sharma, R. L., Kashyap, "Information Routing and Reliability issues in Distributed Sensor Networks”. IEEE Transaction on signal processing. vol, 40, no.12, 1992.

[19] Sajid. Hussain, Abdul. W., Matin, Obidul. Islam, "Genetic Algorithm for Energy Efficient Clusters in Wireless Sensor Networks”. In the Proceedings of 4th International conference on information technology, pp. 147 - 154, 2007.

[20] Jamal. N., Al-Karaki, Raza. Ul.,Mustafa, Ahmed. E., Kamal, "Data aggregation and routing in Wireless Sensor Networks: optimal and heuristic algorithms”. The International Journal of Computer and Telecommunications Networking, vol.53, no.7, pp. $945-960,2009$.

[21] Ki. Sung., Seo, Gyoo. Seok., Choi, "The Genetic Algorithm Based Route Finding Method for Alternative Paths”. In the Proceedings of International conference on systems, Man and cybernetics, vol.3, pp. 2448 - 2453, 1998.

[22] Darrell. Whitley, “A Genetic Algorithm Tutorial”. Journal of statistics and computing, vol.4, pp. 65-85, 1994.

[23] Jarmo. T., Alander, "Genetic Algorithms: An Introduction”. Available From: ftp://ftp.uwasa.fi/cs/report96-1/SCAI06long.pdf.

[24] R. Nallusamy, K. Duraiswamy, D. Ayya., Muthukumar, "Energy Efficient Clustering and Shortest path Routing in Wireless adhoc Sensor Networks using Approximation Algorithms”. Journal of Mathematics and Technology, vol.1, no.1, pp. 161164, 2010.

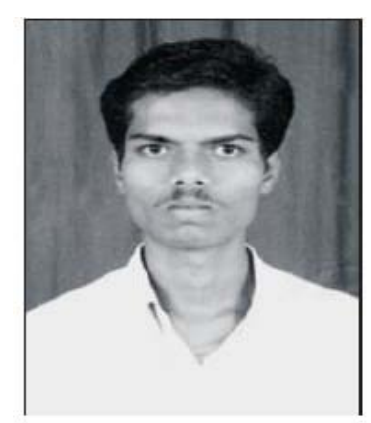

Lokesh B. Bhajantri received $\mathrm{M}$.Tech degree in computer science and Engineering from Basaveshwar Engineering College, Bagalkot, India, in 2005. He is presently working as a Assistant Professor in the Department of Information Science and Engineering, Basaveshwar Engineering college, Bagalkot, India, He has experience of around 10 years in teaching and research. His areas of interest include Distributed Sensor Networks (DSNs), ecommerce, u-commerce, mobile computing and communications, networking protocols, genetic algorithms, applications of agents and real time systems. He has given invited lectures in AICTE sponsored workshops and seminars. He has published chapter in Handbook of Research on Telecommunications Planning and Management for Business, 7 referred international conferences papers and 4 referred international Journals. He is a reviewer of some journals and conferences. He is a member of Board of Studies (BOS) in the Department of Information Science and Engineering, Basaveshwar
Engineering College, Bagalkot, Karnataka, India. He is a member of International Association of Computer Science and Information Technology (IACSIT).

E-mail:

lokeshcse@yahoo.co.in,

lokesh_mb@rediffmail.com

Department of Information Science and Engineering, Basaveshwar Engineering College, Bagalkot

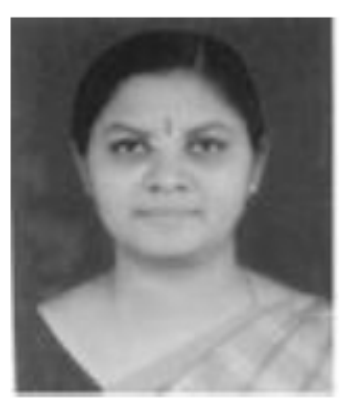

Nalini. $\mathbf{N}$ received Ph.D from Visvesvaraya Technological University, Balgaum, Karnataka, India. She is currently working as Professor and Head of Department of Computer Science and Engineering, Nitte Meenakshi Institute of Technology (NMIT), Bangalore, Karnataka, India. She has experience of around 22 years in teaching and research. She is involved in research of wireless and Distributed Sensor Networks (DSNs), Cloud computing, Cryptography \& Network Security, Genetic Algorithms, and Heuristic Algorithms in Secure Networks. She is an associate editor of Research Journal of Information Technology, Maxwell Scientific Organization. She has many given invited lectures and has conducted several workshops/seminars/conferences. She has published 30 referred Journals and about 50 referred conferences papers. She is a reviewer of many journals and conferences.

E-mail: nalinaniranjan@hotmail.com

Department of Computer Science and Engineering, Nitte Meenakshi Institute of Technology, Bangalore, Karnataka, India 